\title{
The effects of muscle vibration in spasticity, rigidity, and cerebellar disorders ${ }^{1}$
}

\author{
KARL-ERIK HAGBARTH AND GÖRAN EKLUND \\ From the Department of Clinical Neurophysiology, Academic Hospital, Uppsala, Sweden
}

The development of the muscle vibration technique in humans provides a new means for studying the rôle played by muscle afferents in motor control and, furthermore, the technique seems to have diagnostic as well as therapeutic applications. It is now well documented that high frequency mechanical vibration applied to any skeletal muscle in man tends to induce the following reflex response: sustained contraction of the muscle vibrated and simultaneous relaxation of its prime antagonists (Eklund and Hagbarth, 1965, 1966; Hagbarth and Eklund, 1966a; de Gail, Lance, and Neilson, 1966; Lance, de Gail, and Neilson, 1966; Rushworth and Young, 1966). This response has been named TVR (tonic vibration reflex, Eklund and Hagbarth, 1966). Only one kind of muscle afferent unit is known, which, in addition to being sensitive to high frequency vibration, can induce this particular reflex pattern-that is the Ia afferent of the muscle spindles (primary ending), supplying nuclear bag as well as nuclear chain fibres and mediating the phasic as well as the tonic stretch reflex (for references see Jansen, 1962; Granit, 1964; Matthews, 1964; Rushworth, 1964; Eldred and Buchwald, 1967). ${ }^{2}$ There is little doubt that high frequency vibration preferentially excites spindle receptor organs with dynamic properties but the resulting sustained activity in the Ia afferents may to the centres 'resemble' a discharge from spindle organs of the static type. It is still unknown to what extent the vibration reflex in man utilizes the monosynaptic path of the phasic stretch reflex (tendon jerk) or to what extent the same motoneurones are involved in the mediation of the two reflexes (for references see Lang and Vallbo, 1967). However, there is often a striking lack of correspondence between the strength ${ }^{1}$ Based on a lecture given at the international E.M.G.-meeting in Glasgow, 1967. This work was supported by the Swedish Medical Research Council (Project no. K67-14X-02).

'The vibration, as applied on an intact extremity undoubtedly excites many kinds of end-organs including such in the skin, but local anaesthesia of the skin and the subcutaneous tissues underlying the vibrator does not in healthy adults reduce the motor effects of the stimulus (Hagbarth and Eklund, 1966a). of the two reflexes in any particular muscle, and the vibration reflex is comparatively more susceptible to anaesthetic drugs and to neural influences from higher centres. Thus, it was shown in healthy adults that the time course and strength of the vibration reflexes varies not only with the frequency and amplitude of vibration and with the initial state of contraction and length of the muscle vibrated; the reflexes can also be checked by voluntary efforts to resist or counteract the response and they can be influenced by caloric vestibular stimulation, by positional changes of the head, by changes of body temperature (Eklund and Hagbarth, 1966), and by injection of various drugs (de Gail et al., 1966).

Lance et al. (1966) found tonic vibration reflexes of normal strength in the rigid muscles of patients with Parkinson's disease, whereas in patients with cerebellar or upper motor neurone lesions they observed a reduction of the response. Hagbarth and Eklund (1966b) also noted a reduction of the TVR in relaxing hemi- or paraparetic patients. In many of these patients, however, vibration had a marked effect upon voluntary motor performance. The vibration potentiated or reduced voluntary power (and range of movement), depending upon whether the subject tried to contract the muscle vibrated or its antagonist. As judged by force and movement measurements, the potentiating effect usually subsided within a few minutes after the end of vibration but many patients reported that the weakness and spasticity felt less severe for a much longer time (Hagbarth and Eklund, 1966b).

The present investigation is a study of the motor effects of muscle vibration in patients with various types of central motor disorders, in particular those associated with spasticity and rigidity. In 90 patients the effect of muscle vibration was tested not only during rest and relaxation but also during voluntary attempts to move the affected limb. Furthermore, a technique has been developed to measure and test the effect of vibration on different types of abnormal muscle resistance to imposed stretch. 


\section{MATERIAL}

All patients included in the present study were adults, suffering from some kind of central motor disorder. Most of them were referred to us from the neurological or neurosurgical clinic for routine electromyography. Patients with signs of peripheral neuropathy were excluded and so were those with severe contractures. One group consisted of 75 patients with hemi-, quadri-, or paraparesis due to upper motor neurone lesions at various levels of the neuraxis (including tumours, vascular or traumatic lesions, systemic or heredo-degenerative diseases). In this heterogeneous group, the clinical picture showed many variations as regards the degree and distribution of the spasticity and the type of associated sensory disturbances. Some of the patients had very mild symptoms with hardly detectable signs of an upper motor neurone lesion, whereas others had severe motor handicaps. None of the patients had a complete paralysis, however; most of them were ambulatory. The duration of the illness varied from a few weeks up to several years. Most of them received some kind of physical therapy.

Ten patients with Parkinsonism were also examined. Three of them had a hemi-syndrome; all had a more or less marked rigidity and six of them had also an overt tremor. Five patients with cerebellar syndromes constitute the third group. Their motor power was unaffected, the muscle tone was low or normal; they all had ataxic gait and intention tremor and they performed badly in co-ordination tests. Two of them had a hemi-syndrome.

\section{METHODS}

VIBRATION TECHNIQUE The vibrators consist of small eccentrically loaded motors with a cylindrical shape. Rubber bands are used to attach the vibrators transversely over the muscle tendons. The motor $(20 \mathrm{~W})$ rotates with a speed of $150-160 \mathrm{~Hz}$ and produces cyclic vibrations with a peak amplitude of 1.5-2 mm, largely independent of the tension in the rubber bands and the softness of the underlying tissues. The instructions given to the patients during the various tests are described in the 'Results' section.

RECORDING In some of the patients the effect of vibration was evaluated merely by observing the resulting reflex movement or the changes produced in the range of active movements or in the resistance against passive flexion and extension. E.M.G. recordings (with coaxial or surface electrodes) were frequently performed during the tests, however, and potentiometers and strain-gauge dynamometers were used to measure angular joint movements and/or the torques developed by the contracting muscles.

The machine constructed to measure resistance against imposed length changes is similar to that described by Webster (1959). It produces linear motion over a wide range of angular velocities. While the forearm or the leg is carried back and forth at constant speed through, for example, $80^{\circ}$ of passive motion, the angular positional changes and the resulting forces are sensed by the transducers. The signals are fed into a FM tape recorder (PI-6200) from which the stored events are afterwards played back in the form of X-Y plots on an oscilloscope with torque as the ordinate and displacement as the abscissa.

\section{RESULTS}

VIBRATION TESTS DURING REST In these tests the limb to be examined was usually hanging freely or it was supported in a comfortable position. Alternatively, the subject was instructed to keep the limb raised in a defined anti-gravity position. His eyes were closed and he was told to remain passive in the sense that he should not try to counteract or enhance any movements caused by the vibration. Usually his attention was diverted from the test to be performed. In patients with unilateral symptoms two identical vibrators were frequently used and vibration of homologous muscles was started simultaneously on the affected and on the control side. In order to avoid physiological asymmetries in the responses, care was taken to keep the patient's head in midposition and his limbs in similar initial postures (Eklund and Hagbarth, 1966).

Lance et al. (1966) and Hagbarth and Eklund (1966b) stated that the tonic vibration reflexes may be reduced by upper motor neurone lesions. According to our present experience the reflexes are usually weak in paretic muscles acting against spastic antagonists and a sustainedo anti-gravity contraction in these antagonists is not always inhibited by the vibration. In the spastic muscles themselves, however, the reflexes may be reduced, off 0 normal strength, or exaggerated. A study is in progress to find out to what extent this variability depends upon the initial length of the spastic muscle vibrated.

A finding of particular diagnostic interest was that in many hemiparetic patients vibration on the palmar side of the wrist uncovered an abnormal tendency towards pronation. Vibration so applied in a healthy person holding his hand in a supinated position induces a slow finger flexion, eventually combined with a slight pronation and flexion of the hand. In many patients with mild hemiparesis the pronation movement caused by vibration was much stronger than normal, indicating a latent spasticity in the pronator muscles, not easily revealed with ordinary clinical tests.

In many spastic muscles there was a change not only in the strength but also in the time course of the vibration reflex. Thus, as judged by visual inspection and E.M.G. recordings, the sustained response to vibration often started and ceased more abruptly in spastic than in normal muscles. Vibration, suddenly applied to a spastic muscle usually caused an initial jerk-in the E.M.G. appearing as a short burst of electrical activity followed by a silent period of about $100 \mathrm{msec}$. This period was succeeded by a sustained reflex activity which ceased abruptly when vibration stopped. On the whole, the vibration reflexes in human spastic muscles (Fig. 1) often have a time course similar to that described for the soleus muscle of decerebrate cats (Matthews, 1966).

In some patients with severe hemiparesis a sustained reflex contraction appeared not only in the muscle vibrated but also in muscles acting on neighbouring joints. Occasionally the contraction involved also the 
antagonists which are normally inhibited by the vibration. In a few patients with severe upper motor neurone lesions we have even recorded a vibration-induced contraction in the antagonist without concomitant contraction in the muscle underlying the vibrator. Only in some of the patients could such abnormal responses to vibration be explained by a peripheral spread of the vibration stimulus to 'supersensitive' neighbouring muscles.

Vibration on the Achilles tendon in patients with exaggerated tendon jerks in the calf muscles frequently initiated a sustained foot clonus which usually outlasted the vibration. As previously shown, rhythmic contractions of a clonic type may appear also in healthy subjects in response to vibration, especially in the soleus muscle (Hagbarth and Eklund, 1966a).

In the 10 patients with Parkinsonism we found an apparently normal variability between individuals as regards the strength of the vibration reflexes, and there was no constant relation between the degree of rigidity and the strength of the vibration reflex in any particular muscle. Variable results were obtained when recording from rigid antagonists to the muscle vibrated. A vibrationinduced reciprocal inhibition was occasionally seen, but in other instances vibration caused a more widespread increase of rigidity involving also the antagonists. In five of the patients with a Parkinsonian syndrome, vibration on the palmar side of the wrist caused an abnormal pronation movement similar to that seen in spasticity.

In muscles exhibiting tremor it was often hard to evaluate the actual strength of the tonic vibration reflex, especially since the tremor was usually enhanced by vibration. Quite often a tremor appeared in response to vibration, not only in the muscle vibrated and its antago-

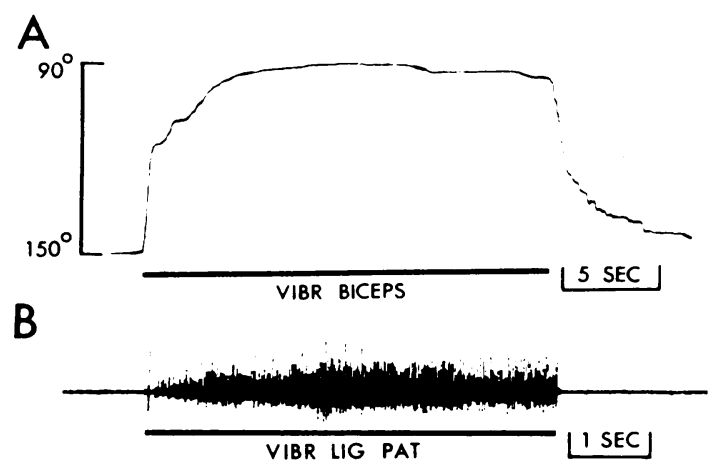

FIG. 1. Two examples of the time course of TVR in patients with spasticity. A shows the resulting movement of the forearm against gravity in response to vibration (bar) on the biceps brachii muscle. B, from another patient, shows the E.M.G. recorded with surface electrodes on the quadriceps muscle. The start of vibration (bar) of the patellar ligament causes a synchronous burst followed by a silent period of about $100 \mathrm{msec}$ duration. Note also the sudden E.M.G. silence when vibration is stopped. (Cf. the more gradual onset and decline of vibration reflexes in healthy adults, e.g. Fig. 6 in Eklund and Hagbarth, 1966.) nist but also in muscles acting at neighbouring joints and even in contralateral limbs.

Weak vibration reflexes could be evoked in the extremity muscles of all three patients with bilateral cerebellar syndromes. Only in one patient with a unilateral syndrome were the reflexes definitely weaker in the affected arm (cf. De Gail et al., 1966; Lance et al., 1966). In another patient with a marked cerebellar syndrome, vibration on the palmar side of the wrist caused a distinct pronation movement.

EFFECT OF VIBRATION ON VOLUNTARY MOVEMENTS lipper motor neurone lesions Many patients with a hemi- or paraparesis could not actively overcome the vibration reflex when trying to make an active joint movement opposing that induced by vibration. In some of them, in particular in those with severe weakness, the effort to make such a movement resulted in paradoxical enhancement of the reflex and thus a movement opposite to that intended. As judged by E.M.G. recordings, others could only with great effort lessen the vibration-induced contraction in a spastic muscle and partly overcome the reciprocal inhibition of the antagonist. This vibrationinduced 'paresis' was often quite pronounced also when the vibration reflex in itself, as tested during rest, was too weak to produce any overt movement. Since the phenomenon occurred also in patients with very mild symptoms of an upper motor neurone lesion, we believe it may serve as an early diagnostic sign. Figure 2 (left half) illustrates an example of a vibration-induced paresis in the knee flexors in a patient with no initial signs of muscle weakness.

On the other hand, vibration applied on a paretic muscle, acting against a spastic antagonist, usually helped the patient to contract the weak muscle and to overcome the spastic resistance in the antagonist. In some patients this potentiating effect of vibration was strong enough to cause a marked enhancement of the active range of movement, in others the effect was weak or negligible. To test the therapeutic value of vibration, 20 patients belonging to the first category received daily vibration treatments, when each attempt to contract the paretic muscle was supported by vibration on the muscle tendon. Following most of these sessions the active range of movement was temporarily enhanced and many patients reported that the weakness and spasticity felt less severe for about 20-30 minutes (cf. Hagbarth and Eklund, 1966b). We still do not know whether repetition of such treatment may have any cumulative effect but some of our patients showed a remarkable motor recovery within a few weeks after the vibration treatment was started.

Parkinsonism and cerebellar disorders No improvement of voluntary motor performance, similar to that occurring in spasticity, was seen when vibrating the muscles of patients with Parkinsonism and cerebellar disorders. Either vibration had no obvious effect on the patient's motor control or it impaired his ability to move. The pronation movement, induced by wrist vibration in some of the patients, was often hard to overcome by voluntary effort. The ataxic patient exhibiting this phenomenon was totally unable to supinate his hand as 


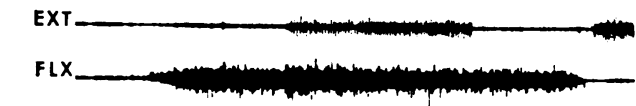

A
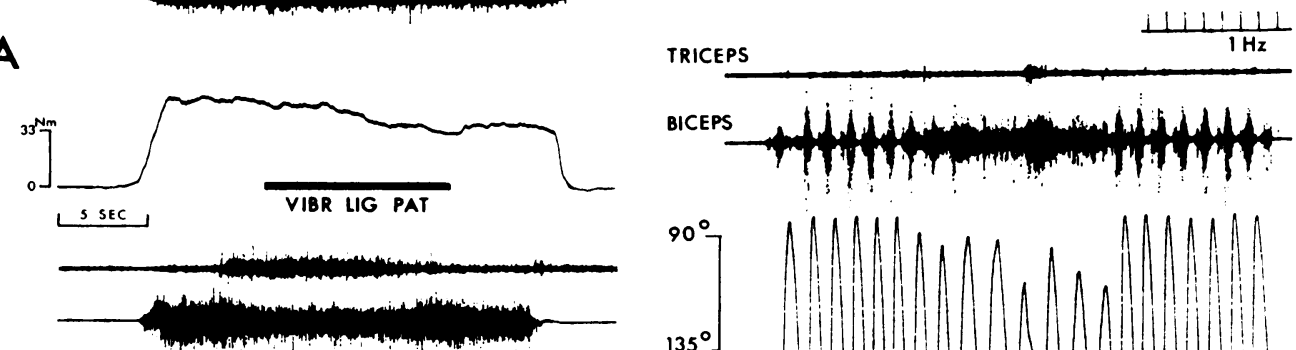

B
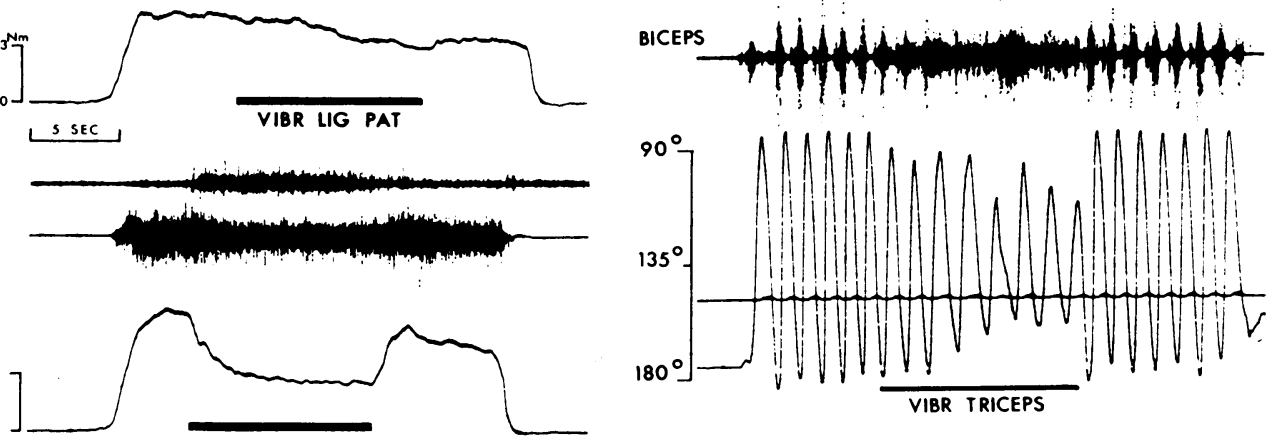

FIG. 2. Two examples of the diagnostic value of vibration. The left part of Fig. 2 is from a patient with very mild signs of an upper motor neurone lesion affecting the left leg. The patient makes a maximal effort to flex his knees (A the right, B the left). The E.M.G.traces are from the quadriceps muscle (EXT) and from the biceps-semitendinosus (FLX). The resulting torques measured isometrically (knee angle $\left.90^{\circ}\right)$ are shown below the E.M.G. traces. Bar indicates vibration of the patellar ligament. In the left limb there was a marked reduction of flexor power during extensor vibration, a temporary 'paresis' which was easily felt by the examiner.

The right part of Fig. 2 is from a patient with mild cerebellar hemiataxia. In standing position he is in- $\mathrm{T}$ structed to perform maximal alternating elbow flexions and extensions on the affected side as fast as possible. The $\mathrm{O}$ resulting movements are recorded below the E.M.G. traces from triceps and biceps. Vibration of the triceps muscle greatly reduces the speed and the amplitude of the movements which are performed awkwardly and with great effort.

long as the wrist vibration continued. As a rule, however, muscle vibration in these patients impaired muscle coordination rather than muscle strength. Thus, in five patients with Parkinsonism and in three with cerebellar disorders, the ability to perform rapid alternating joint movements was greatly reduced by vibration applied to either the flexor or the extensor muscles engaged. E.M.G. recordings during these tests revealed a marked disturbance of the reciprocal control of the muscles (Fig. 2, right part).

EFFECT OF MUSCLE VIBRATION UPON RESISTANCE TO PASSIVE MOVEMENTS Most healthy adults can learn to relax and prevent active muscle resistance against passive slow alternating movements of, for example, the elbow joint. This ability to relax is reduced when the passive movements are performed during continous vibration of the elbow flexors or extensors. The hysteresis diagrams in Fig. 3 show the overall increase of tension produced by the muscle vibrated and the elastic character of the vibration-induced muscle resistance to slow passive stretch. These results confirm that the strength of the vibration reflex tends to increase with increasing passive length of the muscle vibrated (Eklund and Hagbarth, 1966). As the speed of the passive movements is raised, however, the resistance may lose its elastic properties so that, following an initial peak of tension, it declines towards the end of the stretch phase (see Fig. 3).

Variable results have been obtained in preliminary vibration tests performed during passive movements in paretic limbs. The results varied from one patient to the other rather than from one test to the next in the sameo patient. The hysteresis diagrams shown in Fig. 4 are not to be regarded as representative for spastic patients in general; they mainly serve to illustrate the kind of information that can be obtained with this technique. In this patient vibration of the spastic elbow flexors potentiated their elastic resistance to stretch, so that during vibration the tension not only started to rise earlier but also rose more steeply with increasing muscle length. During this test the abnormal extensor resistance (during the flexion phase) remained rather similar to the control. On the other hand, vibration on the elbow extensors not only accentuated their resistance to flexion; it also inhibited the spasticity in the antagonistic flexors. In other patients with a similar degree of muscular hypertonia the spastic resistance increased both in the muscle vibrated and its antagonists; it even happened that the antagonists responded in this way without concomitant changes occurring in the muscle vibrated. At present attempts are being made (Eklund, to be published) to find out to what extent the outcome of these vibration tests in any particular patient can be correlated with the site of the central lesion or to the type and degree of muscular hypertonia.

\section{DISCUSSION}

It seems safe to maintain that the primary endings of the muscle spindles are the receptors mainly 


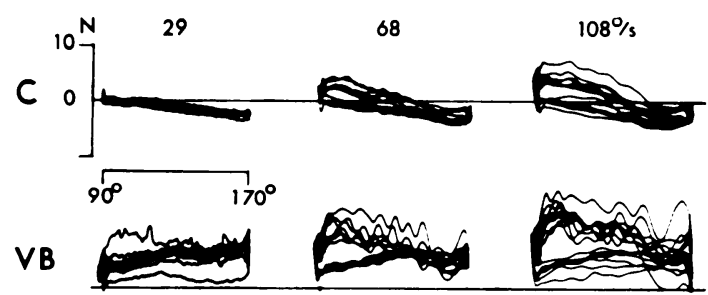

VT



FIG. 3. Resistance to passive alternating movements of the elbow joint in a healthy subject without vibration (C), during vibration of the biceps muscle (VB), and during vibration of the triceps muscle (VT). The vertical scale in each trace shows the resistance to the movements measured in Newton at the wrist $25 \mathrm{~cm}$ from the joint axis. On the horizontal scale movement is from $90^{\circ}$ to $170^{\circ}$. Three velocities $\left(29,68\right.$, and $\left.108^{\circ} / \mathrm{s}\right)$ of movements were used in each case. Each trace consists of 4-6 cycles. The lengthtension interdependence is plotted as hysteresis diagrams in which clockwise loops are traced with upward deflection indicating resistance in the elbow flexors, downward deflection resistance in the extensors. Calibrations only in upper left trace, horizontal line indicates zero tension.
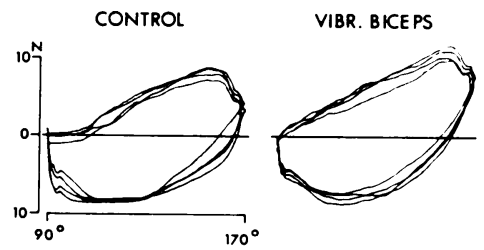

VIBR. TRICEPS

FIG. 4. Traces obtained in a similar way as in Figure 3. Passive movements of the elbow joint in a patient with $a$ marked hypertonus in both elbow flexors and extensors due to amyotrophic lateral sclerosis. The angular velocity was $68 \%$ and thus, these three loops should be compared with the middle column of traces in Figure 3.

responsible for the initiation of the tonic vibration reflexes in healthy adults (Eklund and Hagbarth, 1965, 1966; Hagbarth and Eklund, 1966a). Intense afferent discharge in human muscle afferents was recently recorded in response to vibration (Hagbarth and Vallbo, 1967b) and low intensity tetanic stimulation of muscle afferents-presumably just sufficient to excite only the large Ia afferents from the muscle spindles-may evoke a tonic reflex motor discharge similar to that induced by vibration (Lang and Vallbo, 1967). The question may be raised whether such an intense, sustained activity in stretch reflex afferents, as that artificially induced by vibration, does ever occur 'normally' in man. As judged by recordings from human muscle afferents it may possibly occur during sustained voluntary muscle contractions, whereas passive muscle stretch or local pressure does not induce an afferent activity of similar intensity (Hagbarth and Vallbo, 1967a,b; Vallbo, 1967).

The pattern characterizing the motor response to vibration in healthy adults-autogenic excitation and reciprocal inhibition-was found also in many patients with central motor disorders. However, in some patients with severe spastic paresis this vibration-induced stretch reflex pattern was partially or totally lost; we have seen examples of how the pattern may be totally reversed and how vibrationinduced contractions may appear in muscles acting on neighbouring joints. When trying to explain such abnormal responses to vibration it is important to recognize that the vibration excites many other end organs than the primary spindle endings and that besides evoking specific reflex patterns the vibration may have unspecific 'arousal' motor effects. In some patients autogenic inhibitory reflexes evoked from, for example, secondary spindle endings or Golgi organs may be strong enough to conceal or overcome the vibration-induced tonic stretch reflex. Thus a weak motor response to vibration in, for example, a spastic muscle may be due to an enhancement of some inhibitory reflex rather than to a decrease of the excitatory vibration reflex. Since the various end organs are not equally sensitive to vibration (Bianconi and van der Meulen, 1963; Brown, Engberg, and Matthews, 1967) systematic variations of the vibration frequency in spastic patients may help to elucidate this problem concerning concealed reflexes.

The considerations presented above call for great care in attempts to interprete the outcome of the vibration tests in terms of gamma tonus and sensitivity of primary spindle endings in spastic and rigid patients. Such interpretations are hazardous also because the strength of the tonic stretch reflex depends not only on spindle sensitivity but also on the functional state of the central paths mediating the reflex. The 'permeability' of these paths may in turn depend not only on inter- and motoneurone excitability but also on the level of presynaptic polarization in the muscle afferent terminals (Eccles, 1964; Devanandan, Eccles, and Stenhouse, 1966; Decandia, Provini, and Táboříková, 1967). Direct recordings of the afferent discharges in human muscle nerves (Hagbarth and Vallbo, 1967a, b) may help to clarify the rôle played by the gamma system 
in determining the strength of the vibration reflexes in normal and hypertonic muscles.

Vibration tests performed during voluntary motor acts offer an interesting possibility of analysing the manner in which tonic stretch reflex mechanisms are integrated in the total motor behaviour under normal and abnormal conditions. Spastic patients exhibit a characteristic lack of ability to control the vibration reflexes actively. This disability may be quite evident even when the reflexes in themselves (as tested during rest) are not exaggerated. Thus, the difficulty in controlling the reflexes is a sign in itself, not directly related to the reflex strength. It seems to be of definite diagnostic interest that, even in patients with mild 'pyramidal signs', muscle vibration may produce a paresis of antagonistic muscles. The strength of this vibration-induced paresis in any particular patient may give a hint as to what extent his inherent muscular weakness is a reflex phenomenon-that is, due to an inhibitory Ia inflow from spastic antagonists.

The extent to which vibration potentiates voluntary power in a paretic muscle also seems to vary independently of the actual strength of the vibration reflex as tested during rest. The potentiating effect may be explained by a vibration-induced facilitation of the lower or upper motoneurones engaged in the willed act to contract the muscle, or the will to move may initiate descending impulses which enhance the vibration-induced excitatory inflow-for example, through a gamma sensitization of the spindles. In those cases where vibration potentiates voluntary power it usually also helps the patient to inhibit the spastic resistance in the antagonists and thus the range of movement is increased. It is well known among physical therapists that the stretch reflex can be used to reinforce voluntary motion in patients with upper neurone lesions (Kabat and Knott, 1953). The vibration technique, however, has definite advantages as compared with ordinary stretching of the muscle against active resistance. Thus, it requires little effort from the physical therapist and in our experience it reinforces voluntary motion more effectively. The vibrators can easily be applied on paretic muscles and be controlled by the patient himself. Two 'electrophysiological bandages' have recently been constructed. One consists of two 'antagonistic' thigh vibrators controlled by heel switches and aiding the patient in respectively the swing and stance phase of walking. In the other 'bandage' the vibrators are controlled by E.M.G. signals from the muscles to be stimulated (Hedberg, Oldberg, and Tove, 1967). The clinical applicability of such devices is being investigated.

Some of the observations described in the present reports require further analysis before any serious attempts can be made to explain them in neurophy siological terms. This applies to, for example, theo disabling effect of vibration upon motor performance in some patients with rigidity and cerebellar syn-s dromes. Some of the patients showed little initia $\overline{6}$ disability to perform alternating movements but as soon as the vibration started their disability to coordinate became pronounced. We believe that? certain types of latent coordination deficiency and also latent tremor may be revealed by the vibration technique.

The strength of the tonic vibration reflex in? healthy adults tends to increase with increasinge muscle length, thus yielding an elastic resistance to $\overline{\bar{D}}$. slow stretch, similar to the type of resistance en-त्ब countered in many spastic muscles. The main reason why muscle stretch enhances the vibration reflex is ${ }^{\circ}$ probably that an elongation of the muscle spindles $\overrightarrow{0}$ makes them more susceptible to the vibrationstimulus: it has been shown both in cat and mancल that the vibration-induced afferent discharge in creases with increasing muscle length (Granit and Henatsch, 1956; Hagbarth and Vallbo, 1967b; Brown et al., 1967). A sustained, length-dependent: discharge in the Ia afferents, similar to that artifie ally induced by vibration in normal subjects, m\&y well occur 'spontaneously' in patients where theㄹ응 is an abnormal release of the static fusimotor syste. It must be recognized, however, that there are some important dissimilarities between the spastic staffe on the one hand and the motor effect of muse -1 vibration in healthy adults on the other. Firstly, healthy adult can voluntarily overcome the motor ${ }^{\infty}$ effect of vibration and, secondly, the phasic stretch reflex, as a rule enhanced in spasticity, tends to bes suppressed during vibration in healthy adultsō (Eklund and Hagbarth, 1965; Hagbarth and\%ँ Eklund, 1966a; de Gail et al., 1966; Lance et al., $\stackrel{\mathbb{Q}}{\circ}$ 1966, Rushworth and Young, 1966).

\section{SUMMARY}

This report is based upon a study involving $75 \frac{\mathrm{J}}{0}$ patients with upper motor neurone lesions, $10 \%$ patients with Parkinsonism, and five with cerebellar syndromes. The motor effects of high frequency mechanical muscle vibration (about $150 \mathrm{~Hz}$ ) was studied (1) during rest and relaxation, (2) duringo voluntary attempts to activate the muscles and to $D$ perform alternating movements, and (3) duringo passive movements performed to test muscle resistance to imposed stretch. The results show that noto only the strength, but also the time course of the N vibration reflex may be different in spastic as com- N pared with normal muscles. Another common 
finding in spastic patients is that vibration potentiates or reduces voluntary power (and range of movement) depending upon whether the subject tries to contract the muscle vibrated or its antagonist. The potentiating effect upon active range of movement partly depends upon a vibration-induced reciprocal inhibition of the spastic resistance in the antagonists. Co-contractions in antagonists and other abnormal vibration reflex patterns were observed in some of the spastic patients. In patients with Parkinsonism, the vibration reflexes are often of normal strength but vibration increases the tremor and it may also impair the patient's ability to perform alternating movements. A similar vibration-induced disability to perform fast alternating movements was observed also in patients with cerebellar disorders, where the vibration reflexes (as tested during rest) were either of normal strength or reduced. Diagnostic and therapeutic aspects of muscle vibration are discussed.

We are in particular indebted to our colleagues in the neurological department (Head Professor K. A. Ekbom) for permission to perform vibration tests on many of their patients.

\section{REFERENCES}

Bianconi, R., and van der Meulen, J. P. (1963). The response to vibration of the end organs of mammalian muscle spindles. $J$. Neurophysiol., 26, 177-190.

Brown, M. C., Engberg, I., and Matthews, P. B. C. (1967). The relative sensitivity to vibration of muscle receptors of the cat. J. Physiol. (Lond.), 192, 773-800.

Decandia, M., Provini, L., and Tábořiková, H. (1967). Presynaptic inhibition of the monosynaptic reflex following the stimulation of nerves to extensor muscles of the ankle. Exp. Brain Res., 4, 34-42.

de Gail, P., Lance, J. W., and Neilson, P. D. (1966). Differential effects on tonic and phasic reflex mechanisms produced by vibration of muscles in man. J. Neurol. Neurosurg. Psychiat., 29, 1-11.

Devanandan, M. S., Eccles, R. M., and Stenhouse, D. (1966). Presynaptic inhibition evoked by muscle contraction. J. Physiol. (Lond.), 185, 471-485.
Eccles, J. C. (1964). The Physiology of Synapses, pp . 98-100, 220234. Springer, Berlin.

Eklund, G., and Hagbarth, K.-E. (1965). Motor effects of vibratory muscle stimuli in man. Electroenceph. clin. Neurophysiol., 19, 619.

-, (1966). Normal variability of tonic vibration reflexes in man. Exp. Neurol., 16, 80-92.

Eldred, E., and Buchwald, J. (1967). Central nervous system: motor mechanisms. Ann. Rev. Physiol., 29, 573-606.

Granit, R. (1964). The gamma $(\gamma)$ loop in the mediation of muscle tone. Clin. Pharmacol. Ther., 5, 837-847.

- , and Henatsch, H. D. (1956). Gamma control of dynamic properties of muscle spindles. J. Neurophysiol., 19, 356-366.

Hagbarth, K.-E., and Eklund, G. (1966a). Motor effects of vibratory muscle stimuli in man, in Proceedings of the First Nobel Symposium, pp. 177-186. Edited by R. Granit. Almqvist and Wiksell, Stockholm.

— - (1966b). Tonic vibration reflexes (TVR) in spasticity. Brain Res., 2, 201-203.

_ and Vallbo, $\AA$. B. (1967a). Mechanoreceptor activity recorded percutaneously with semi-microelectrodes in human peripheral nerves. Acta physiol. scand., 69, 121-122.

- 1 (1967b). Afferent response to mechanical stimulation of muscle receptors in man. Acta Soc. Med. upsalien., 72, 102-104

Hedberg, L. A., Oldberg, B., and Tove, P. A. (1967). EMG-controlled muscle vibrators to aid mobility in spastic paresis, in Digest of the 7th International Conference on Medical and Biological Engineering, p. 197. Edited by B. Jacobson. Almqvist and Wiksell, Stockholm.

Jansen, J. K. S. (1962). Spasticity-functional aspects. Acta neurol. scand., 38, suppl. 3, 41-51.

Kabat, H., and Knott, M. (1953). Proprioceptive facilitation technics for treatment of paralysis. Phys. Ther. Rev., 33, 53-64.

Lance, J. W., de Gail, P., and Neilson, P. D. (1966). Tonic and phasic spinal cord mechanisms in man, J. Neurol. Neurosurg. Psychiat., 29, 535-544.

Lang, A. H., and Vallbo, Å. B. (1967). Motoneurone activation by low intensity tetanic stimulation of muscle afferents in man. Exp. Neurol., 18, 383-391.

Matthews, P. B. C. (1964). Muscle spindles and their motor control. Physiol. Rev., 44, 219-288.

- (1966). The reflex excitation of the soleus muscle of the decerebrate cat caused by vibration applied to its tendon. J. Physiol. (Lond.), 184, 450-472.

Rushworth, G. (1964). Some aspects of the pathophysiology of spasticity and rigidity. Clin. Pharmacol. Ther., 5, 828-836.

- , and Young R. R. (1966). The effect of vibration on tonic and phasic reflexes in man. J. Physiol. (Lond.), 185, 63P-64P.

Vallbo, A. B. (1967). Impulse patterns in a muscle nerve during voluntary contraction in man. Acta physiol. scand., 69, 123-124.

Webster, D. D. (1959). A method of measuring the dynamic characteristics of muscle rigidity, strength, and tremor in the upper extremity. IRE. Trans. med. Electronics, 3, 159-164. 